\title{
Study of cracks in concrete target under deep penetration by a projectile
}

\author{
Cheng $\mathbf{W u} \mathbf{u}^{\mathrm{a}}$ \\ Wenbin Li* \\ Xiaojun Shen ${ }^{b}$ \\ Wenjin Yao ${ }^{\mathrm{a}}$ \\ a Ministerial Key Laboratory of ZNDY, Nanjing University of Science and Technology, Nanjing 210094, China. \\ E-mail: qqqwucheng@163.com, Iwb2000cn@njust.edu.cn, njyaowj@163.com \\ b Beijing Special Electrical and Mechanical Research Institute, Beijing 100012, China. E-mail: SXJ_1959@126.com \\ * Corresponding author
}

http://dx.doi.org/10.1590/1679-78255382

\begin{abstract}
A large number of cracks occur in concrete structure after projectile penetration. The study of crack distribution and propagation process is the basis of damage assessment, structural repair and high dynamic fracture mechanism. In this paper, wire saw cutting, X-ray computed tomography (X-CT) and mechanical property testing of damaged concrete were carried out on deep penetration targets. According to X-CT images of radial core samples in target and the mechanical properties of damaged concrete, the crack distribution in the target was divided into a plastic damage zone and a brittle damage zone, and the microcracks only existed in the plastic damage zone. The initial growth process of three-dimensional cracks was obtained by X-CT images of axial core samples in target, that is, the cracks in the pure mortar target gradually developed from the radial direction to the tangential direction, and the target containing coarse aggregate directly formed tangential cracks. The propagation progress of cracks was obtained through the target section, that is, the tangential cracks bent in the pure mortar target, and were relatively straight in the target containing coarse aggregate. A crack propagation model was established, and the tangential crack formula and the crack propagation velocity were obtained.
\end{abstract}

\section{Keywords}

Crack; Penetration; Concrete; Projectile; Damage

\section{INTRODUCTION}

Because concrete materials have tensile brittleness, a large number of cracks can occur after concrete structure is struck by a projectile, resulting in a decrease in the resistance to multiple penetrations and the bearing capacity. For structures close to water (such as dams) in cold regions, the appearance of cracks causes the structure to undergo secondary damage due to frost heave. The study of crack distribution is helpful to evaluate the damage degree in different locations, and lays a foundation for damage assessment and structural repair. The study of crack propagation can give the initiation and propagation progress of the crack, and lays a foundation for the development of high dynamic fracture mechanism.

Preliminary research has been previously conducted on target cracks subsequent to penetration. A number of experiments and numerical simulations have been carried out on the surface cracks of various target and the internal cracks of the thin target and the shallow penetration target. The surface of the target appears crater and radiation 
cracks (Frew et al., 2006; Lai et al., 2015); the thin targets that are not perforated appears tensile scabbing on their back (Li et al., 2005; Sovják et al., 2015); the perforated thin target appears shear plug (Pereira et al., 2018; Tai, 2009); and the shallow penetrated target appears spherical radiation cracks inward (Forquin et al., 2008; Forquin et al., 2015). The study of cracks in deep penetration target is mainly based on two-dimensional numerical simulation (Beissel and Johnson, 2000; Lian et al., 2010), but there are few experimental studies, and it is necessary to demonstrate the basic characteristics of cracks in deep penetration target via experimentation. The target section can show the overall distribution of the cracks, and for the deep penetration target, it is usually obtained by prying open the cracks (Wan et al., 2016; Wu et al., 2012); however, this method results in further crack expansion. The further expansion of cracks can be avoided by wire saw cutting, and the smooth section of various positions and directions can be obtained. The crack distribution is asymmetric, and the three-dimensional spatial distribution of the cracks surfaces can be obtained by Xray computed tomography (X-CT). Cracks have significant changes in the mechanical properties of concrete, so it is necessary to test the mechanical properties of damaged concrete at different locations.

Crack propagation velocity is an important property of crack. At high strain rates, the crack propagation velocity of concrete approaches a constant value (Pyo et al., 2016; Pyo and El-Tawil, 2013). Based on the Griffith fracture theory, Roberts and Wells obtained the ultimate crack velocity, which is 0.38 times the elastic longitudinal wave velocity $C_{L}$ (Kanninen and Popelar, 1987). However, the experimental result differs from the above theory. The ultimate crack velocities of Homalite-100, glass, Plexiglas and concrete are $0.23 C_{L}, 0.30 C_{L}, 0.35 C_{L}$ and $0.41 C_{L}$ (Forquin and Hild, 2010; Zhao and Xiao, 2013). Because the mechanical properties of concrete are complicated, it is difficult to accurately predict the ultimate crack velocity based on the mechanical property-based theory, so it is necessary to develop a crack propagation theory based on crack propagation results.

In the present study, wire saw cutting, X-CT and mechanical property testing of the damaged concrete were carried out for the deep penetration target. The corresponding relationship between crack distribution and concrete damage was analyzed by local mechanical properties and X-CT images of the radial core sample in the damaged target. The initiation and propagation progress of crack was analyzed by X-CT images of the axial core sample and cracks in the target section. Based on these results, a crack propagation theory model was established, that could explain the bending of tangential cracks and calculate the crack propagation velocity.

\section{EXPERIMENTAL PROCESS}

\subsection{Projectile}

The projectile (Fig. 1) consisted of a solid body and an obturator ring with the total mass of $0.976 \mathrm{~kg}$. The mass of the obturator ring was $0.0072 \mathrm{~kg}$. The radius of the projectile $r_{0}$ was $14.95 \mathrm{~mm}$, the projectile length-to-diameter ratio was 7 , and the caliber-radius-head was 4 . The body material was $35 \mathrm{CrMnSiA}$ structural alloy steel, and the Rockwell hardness was $44 \mathrm{HRC}$ (Corresponding tensile strength is $1400 \mathrm{MPa}$ ). The obturator ring was connected to the projectile tail by a thread, and its material was monomer casting (MC) nylon.

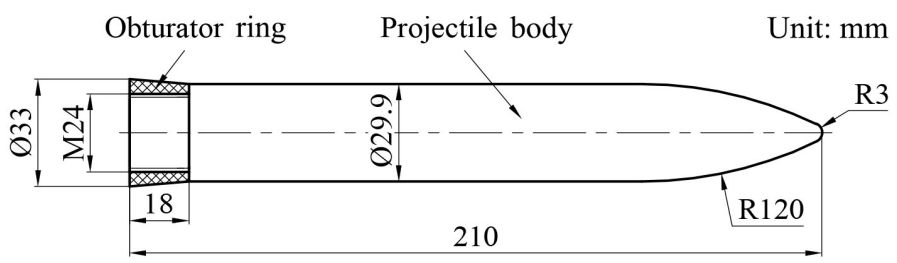

Figure 1: Structure and size of projectile

\subsection{Concrete target}

The concrete mix proportion and basic performance parameters are given in Table 1 . The $\mathrm{c} 1$ was pure mortar, and the c3 contained coarse aggregate. The strength grade of the cement was 32.5R. The fine aggregate was quartz sand. The coarse aggregate was crushed limestone, its natural drying density was $2712 \mathrm{~kg} / \mathrm{m}^{3}$, and the maximum size of the coarse aggregate was $31.5 \mathrm{~mm}$. The uniaxial compressive strength of concrete was tested approximately 4 months after the pouring date by $150 \mathrm{~mm}$ cube. The elastic modulus and Poisson ratio were tested approximately 8 months after the pouring date by samples with a diameter of $50 \mathrm{~mm}$ and a length of $100 \mathrm{~mm}$. 
Table 1: Concrete mix proportion and basic performance parameters

\begin{tabular}{|c|c|c|c|c|c|c|c|c|c|}
\hline \multirow[b]{2}{*}{$\begin{array}{l}\text { Concret } \\
\text { e No. }\end{array}$} & \multicolumn{5}{|c|}{ Mass mix proportion } & \multicolumn{4}{|c|}{ Basic performance } \\
\hline & Cement & $\begin{array}{c}\text { Wate } \\
r\end{array}$ & $\begin{array}{c}\text { Fine } \\
\text { aggregate }\end{array}$ & $\begin{array}{c}\text { Coarse } \\
\text { aggregate }\end{array}$ & $\begin{array}{l}\text { Density } \\
\left(\mathrm{kg} / \mathrm{m}^{3}\right)\end{array}$ & $\begin{array}{c}\text { Uniaxial } \\
\text { compressive } \\
\text { strength (MPa) }\end{array}$ & $\begin{array}{c}\text { Elastic } \\
\text { modulus } \\
E(\mathrm{GPa})\end{array}$ & $\begin{array}{c}\text { Poisson } \\
\text { ratio } \\
v\end{array}$ & $\begin{array}{c}\text { Elastic } \\
\text { longitudinal wave } \\
\text { velocity } C_{L}\end{array}$ \\
\hline c1 & 1 & 0.4 & 1.45 & 0 & 2114 & 38 & 14.1 & 0.106 & 2583 \\
\hline c3 & & & & 2.49 & 2356 & 45 & 25.5 & 0.157 & 3290 \\
\hline
\end{tabular}

The concrete target consists of a $3 \mathrm{~mm}$ thick Q235 steel cylinder and poured concrete. The two targets corresponding to concrete c1 were labeled c1-2 and c1-4, and targets corresponding to c3 were labeled c3-2 and c3-3. The pure mortar target and the target containing coarse aggregate have diameters of $0.9 \mathrm{~m}$ and lengths of $1.45 \mathrm{~m}$ and $1.50 \mathrm{~m}$, respectively.

\subsection{Experiment procedure}

The experiment procedure is shown in Fig. 2. First, the projectile penetrated the concrete target at the initial velocity $V_{0}$, and then cutting the target after penetrated, and finally the core sample was taken on the cutting surface. Among them, the obtained core sample was used for X-CT and mechanical test. The origin of the cylindrical coordinate system is based on the impact point of the target; the axial coordinate is $z$ (Fig. 2a), and the radial coordinate is $r$ (Fig. 2b). The penetration depth of the projectile is $P$ (Fig. 2c).

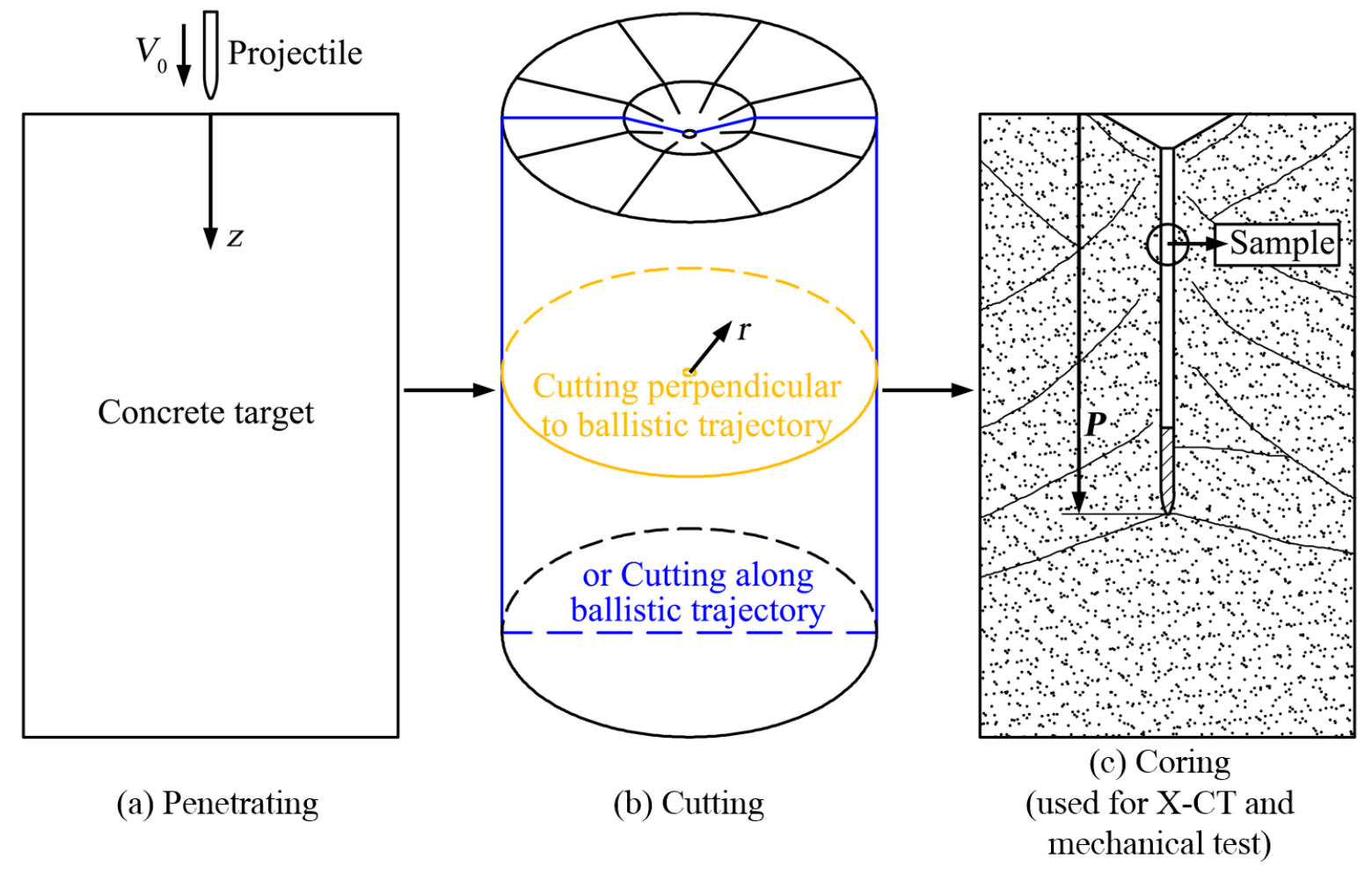

Figure 2: Schematic diagram of the experimental procedure

\subsubsection{Projectile penetrating concrete (Wu et al., 2018)}

The projectiles were launched by a $30 \mathrm{~mm}$ smoothbore gun. The projectile produces a negligible attack angle and penetrates the target with initial velocity $V_{0} \approx 650 \mathrm{~m} / \mathrm{s}$. After penetration, the nose erosion and the deformation of the projectile body are negligible. The tests were performed approximately 3 months after pouring.

\subsubsection{Cutting damaged target by wire saw}

Wire saw cutting was performed on the penetrated target, and smooth sections were obtained (Fig. 3). The scheme is as shown in Table 2. The clear crack images were obtained after watering and drying (Fig. 3b). 


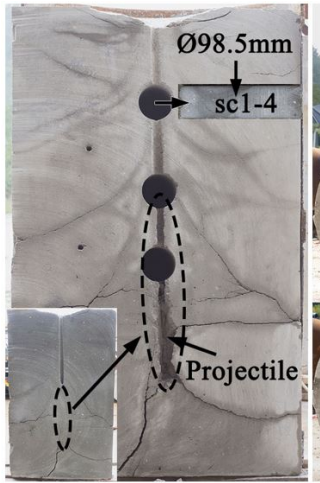

(a) Target $\mathrm{cl}-4$ ( $V_{0}=649 \mathrm{~m} / \mathrm{s}$, $P=1.12 \mathrm{~m}$ )

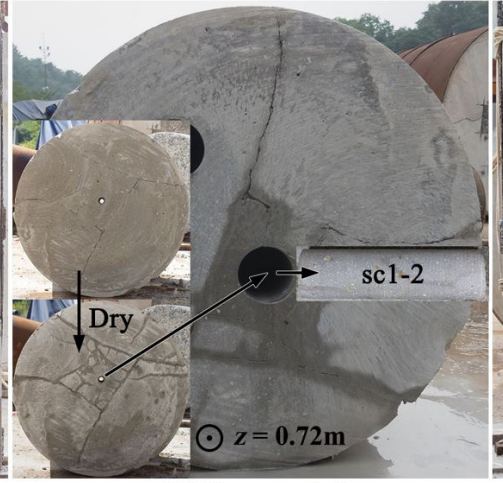

(b) Target $\mathrm{c} 1-2$

( $V_{0}=641 \mathrm{~m} / \mathrm{s}$,

$P=1.03 \mathrm{~m}$ )

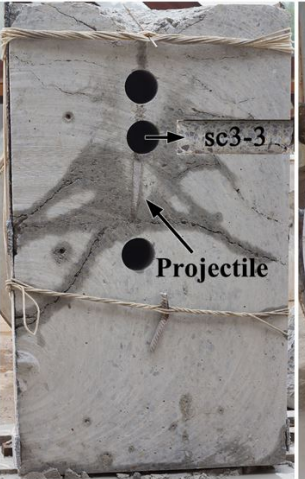

(c) Target c3-3

$\left(V_{0}=650 \mathrm{~m} / \mathrm{s}, P=0.702\right.$

$\mathrm{m})$



(d) Target c3-2 ( $V_{0}=653 \mathrm{~m} / \mathrm{s}$, $P=0.659 \mathrm{~m})$

Figure 3: Wire saw cutting the target after penetrated

Table 2: Scheme of wire saw cutting

\begin{tabular}{cccc}
\hline Target No. & Concrete No. & Target dimension $(\mathrm{m})$ & Cut direction \\
\hline c1-4 & c1 & $\varnothing 0.9 * 1.45$ & Along ballistic trajectory \\
c1-2 & c1 & $\varnothing 0.9 * 1.45$ & Perpendicular to ballistic trajectory \\
c3-3 & c3 & $\varnothing 0.9 * 1.50$ & Along ballistic trajectory \\
c3-2 & c3 & $\varnothing 0.9 * 1.50$ & Perpendicular to ballistic trajectory \\
\hline
\end{tabular}

For target c1-4, the ballistic trajectory was nearly a straight line, but the ballistic trajectory was not easy to cut; consequently, the section surface exhibited a gradual deviation, and the final deviation from the projectile surface was $40 \mathrm{~mm}$; a hammer was employed to knock out the remaining ballistic trajectory (Fig. 3a).

\subsubsection{X-CT of damaged target}

Core samples were taken from the target section (Fig. 3), and the diameter of the core sample was $98.5 \mathrm{~mm}$; the internal pores and cracks in the concrete were observed by X-CT.

\subsubsection{Mechanical properties of damaged concrete}

Concrete cubes with side length of approximately $20 \mathrm{~mm}$ were acquired at different locations of core sample sc1-4 (Fig. 4), and their densities, elastic moduli and uniaxial compressive strengths were tested. Macrocracks were avoided while cutting the core sample. The test date was approximately 5 months after pouring.

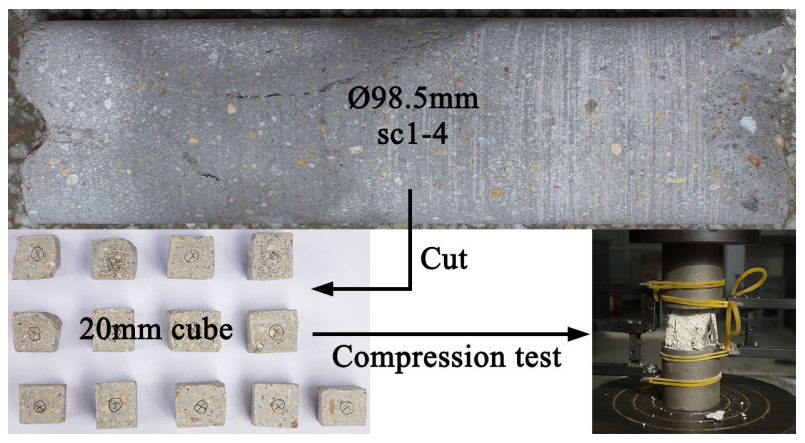

Figure 4: Mechanical properties of damaged concrete

The maximum coarse aggregate size of c3 was $31.5 \mathrm{~mm}$, so c3 is unsuitable for the $20 \mathrm{~mm}$ cube test. Here, only sc1-4 was tested. 


\section{EXPERIMENT RESULTS AND ANALYSIS}

\subsection{Crack distribution}

\subsubsection{Mechanical properties of the damaged concrete}

The density $\rho_{d}$, elastic modulus $E$, and uniaxial compressive strength $f_{c 20}$ of $20 \mathrm{~mm}$ cube (taken from core sample sc1-4) are shown in Fig. 5 . A boundary of $r / r_{0}=1$ indicates that the mortar was close to the projectile surface; compared with the undamaged physical quantities, the saturated density increased by $2.1 \%$, the uniaxial compressive strength decreased by $63.2 \%$, and the elastic modulus decreased by $80.0 \%$. To avoid damage due to machining, the grinding times of the specimen were reduced. However, the stiffness results of the small specimens were sensitive to planeness, which led to a large variation of the elastic modulus.

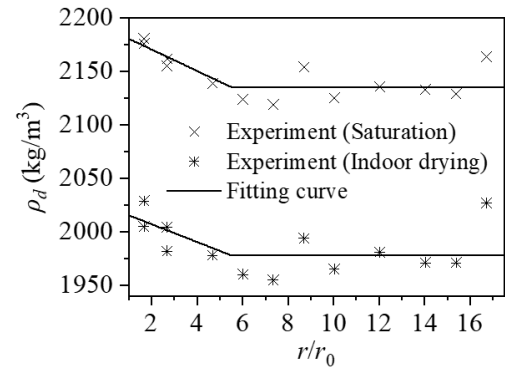

(a) Density

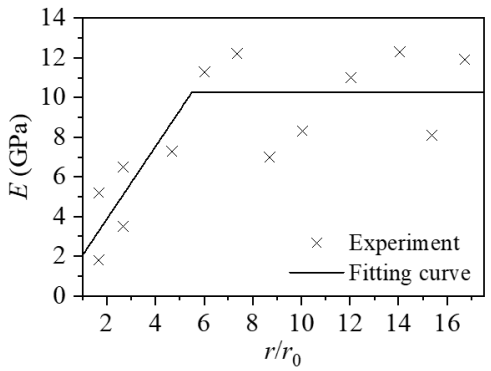

(b) Elastic modulus

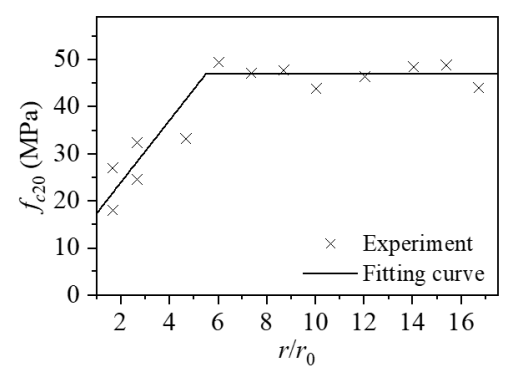

(c) Uniaxial compressive strength

Figure 5: Mechanical properties of the $20 \mathrm{~mm}$ cube taken from core sample sc1-4

The transition boundaries of the density, elastic modulus and uniaxial compressive strength were the same, i.e., $r / r_{0}=5.5$. The mechanical properties within the boundary changed greatly, indicating that plastic compression had taken place overall. The mechanical properties outside the boundary were not affected, indicating that the macrocracks were locally extended.

\subsubsection{X-CT image}

X-CT images of core sample gave the distribution of microcracks and macrocracks, as shown in Fig. 6. The distribution of cracks was divided into a plastic damage zone and a brittle damage zone. The concrete around the ballistics contained microcracks and the number of pores decreased, which was plastic damage zone. Macrocracks appeared in the zone far from the ballistics, which was brittle damage zone. The white dotted line is the boundary between the plastic damage zone and the brittle damage zone.

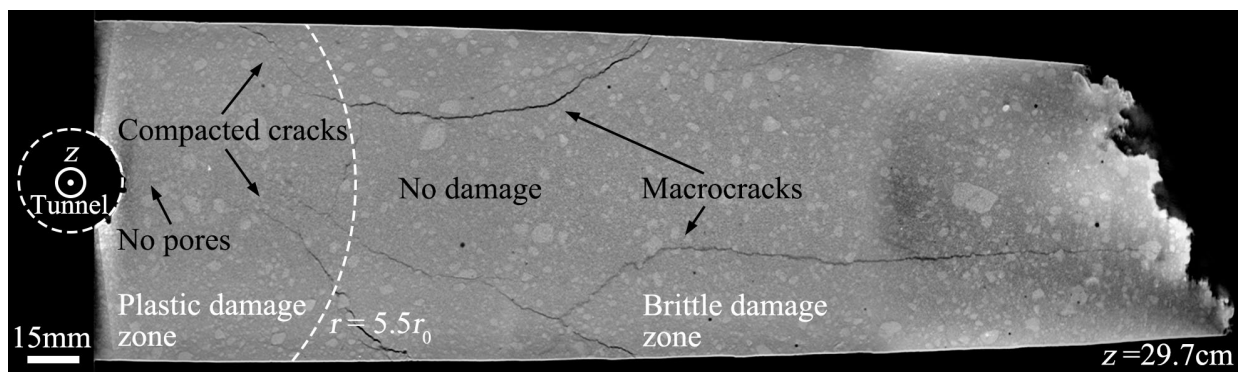

Figure 6: X-CT image of core sample sc1-4

There were no microcracks in the brittle damage zone. In the brittle damage zone, the macrocracks were extended by the concentrated stress, and then the stress of the material near the macrocracks was significantly reduced; thus, no microcracks were generated. That was, macrocracks propagated locally, which was consistent with the conclusion of Section 3.1.1.

There were microcracks in the plastic damage zone. During penetration, the propagation distance of the crack was large, so the target formed extensive brittle damage. As penetration progressed, the projectile nose passed through the concrete, which was already brittlely damaged, causing macrocracks near the projectile nose to be compressed into microcracks. That was, the microcracks in the plastic damage zone were transformed by macrocracks. 
The coarse aggregate had a significant effect on the cracks, as shown in Fig. 7. Coarse aggregate had a higher brittleness than mortar, and so part of the coarse aggregate in the plastic damage zone cracked under compressive stress. For the brittle damaged zone, the randomly distributed coarse aggregate caused the macrocracks to become serrated. The addition of coarse aggregate reduced the compressibility of concrete, increases the intensity of stress wave, resulting in an increase in crack width; the maximum crack width in the pure mortar sample was approximately $0.4 \mathrm{~mm}$, and the maximum width was approximately $1 \mathrm{~mm}$ after adding coarse aggregate.

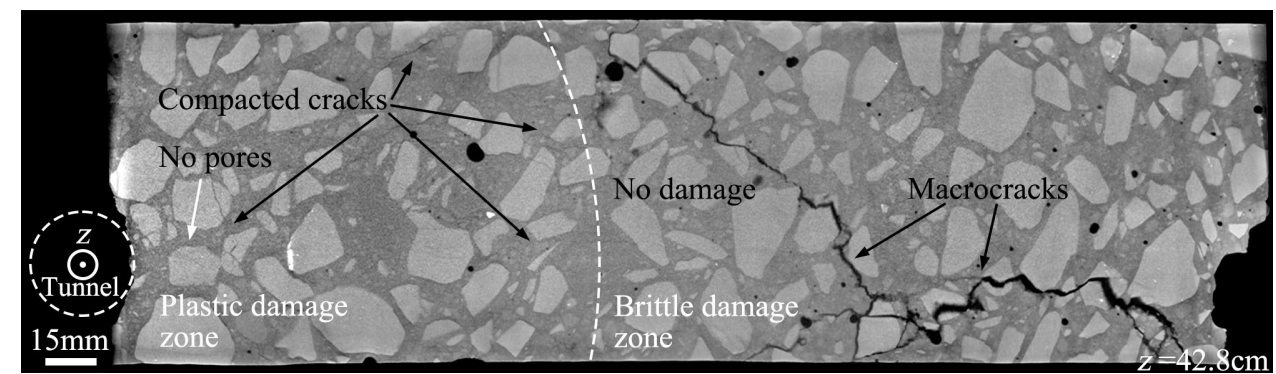

Figure 7: X-CT image of core sample sc3-3

\subsection{Crack propagation process}

Cracks propagated outwards from the vicinity of the ballistic trajectory, and cracks interacted with each other during propagation. The propagation process and law can be determined from the crack distribution. The crack surface on the $\mathrm{rOz}$ plane was defined as a radial crack, and the crack surface perpendicular to the $\mathrm{rOz}$ plane was defined as a tangential crack.

\subsubsection{Crack initiation}

The two-dimensional contour map of the three-dimensional crack surface was obtained by the X-CT images of the axial core sample in the target, and the initial process of the crack surface was obtained through the interactive relationship.

The results from the core sample sc1-2 are shown in Fig. 8. Black lines were used to trace the cracks in the X-CT images to enhance their visibility. The initial direction of cracks $1 \sim 4$ was close to the radial direction, and it became gradually tangential during the outward propagation of the cracks. During the propagation process, cracks 1 and 2 merged, and crack 3 prevented the propagation of crack 4.



Figure 8: Crack contour map of core sample sc1-2

The results from core sample sc3-2 are shown in Fig. 9. The obturator ring at the end of the projectile had a low density, and it is not visible in the image. The metal artifact was formed around the projectile that reduced the sharpness of the image, but it did not affect the overall image. The starting position of the macrocrack was far from the ballistic trajectory, and the radial crack did not form. The macrocrack directly propagated outward in the tangential direction. During the propagation process, crack 1 prevented the propagation of crack 2. 



Figure 9: Crack contour map of core sample sc3-2

\subsubsection{Crack propagation progress}

The cracks in the pure mortar target are shown in Figs. 10 and 11. Among them, the section of Fig. 10a is the r $0 z$ plane, and the section of Fig. 11a is perpendicular to the $z$-axis. To observe the cylindrical surface of the target, the steel plate was removed. The points in the figure represent cracks. The color points are numbered, while the unnumbered and unclear cracks are characterized by smaller black dots. Black dashed lines indicate the cutting position of the uncut target, as shown in Figs. 10b 10e. The direction of the crack surface can be determined by utilizing views with different perspectives for the same crack. For example, cracks 2 and 4 can be identified as tangential cracks by Figs. 10a and 10b, and cracks 18 and 25 can be identified as radial cracks by Fig. 10b and $10 \mathrm{~d}$. The intersections of cracks can be used to determine the timing of arrival at the intersection. For example, crack 6 turns at the intersections of cracks 5 and 6 (Fig. 10a), and it can consequently be determined that crack 5 reached the intersection before crack 6 .
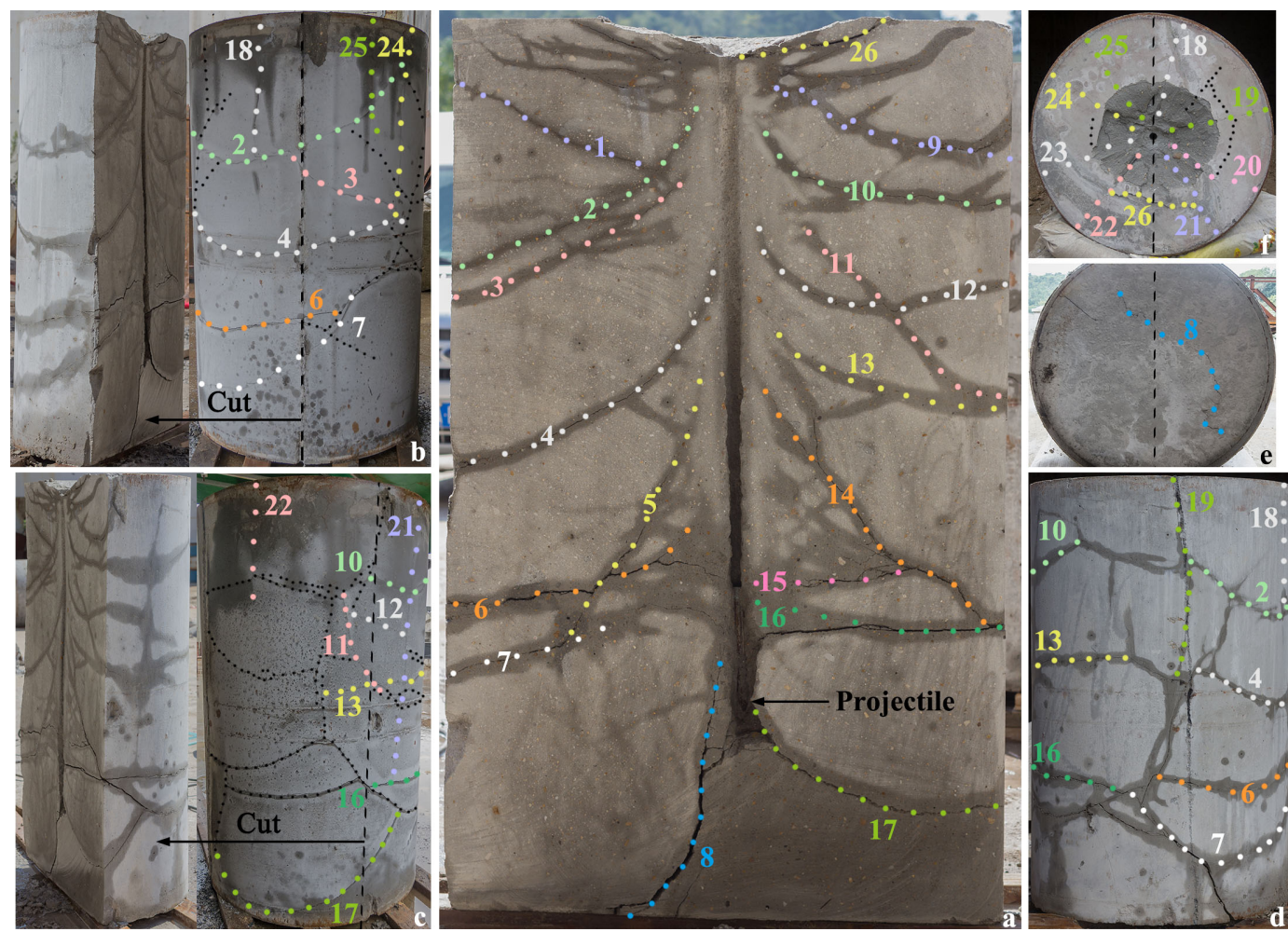

Figure 10: Cracks in target c1-4 ( $\left.V_{0}=649 \mathrm{~m} / \mathrm{s}, P=1.12 \mathrm{~m}\right)$, (a) Section, (b) $(\mathrm{d})$ Side surface, (e) Rear surface, (f) Impact surface 

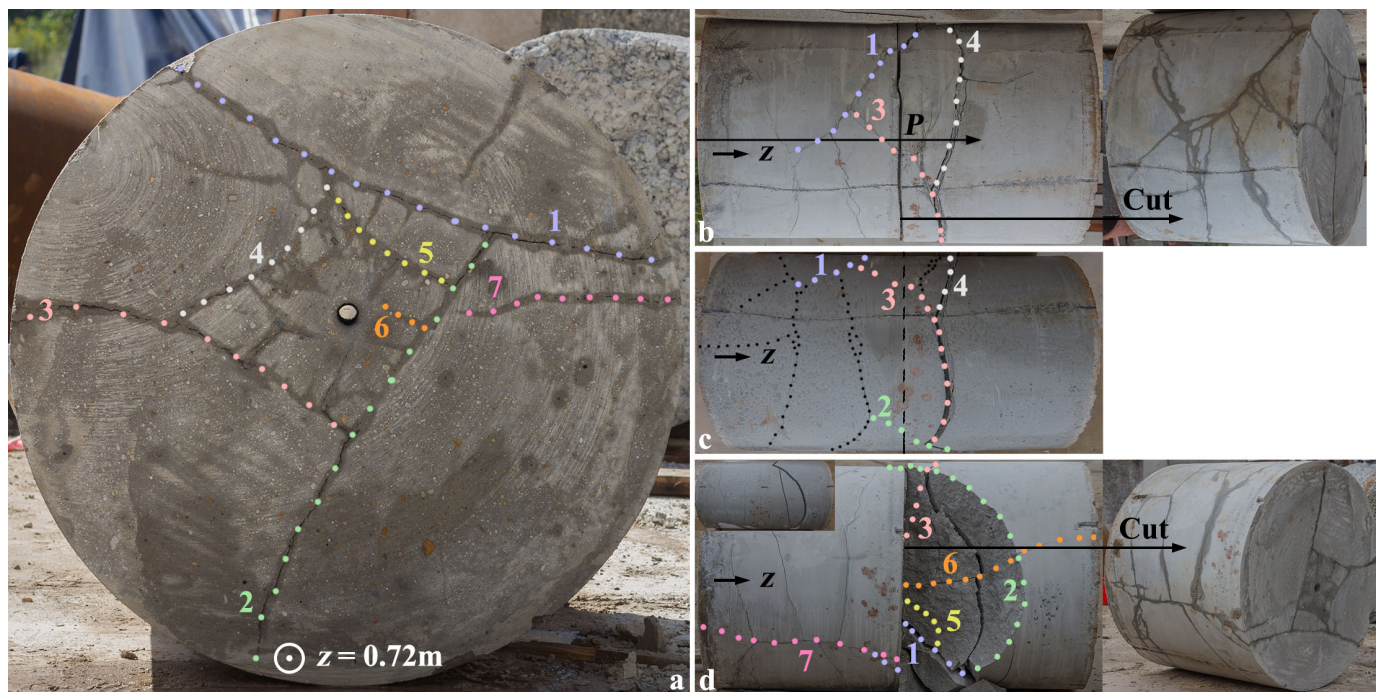

Figure 11: Cracks in target c1-2 $\left(V_{0}=641 \mathrm{~m} / \mathrm{s}, P=1.03 \mathrm{~m}\right)$, (a) Section, (b) (d) Side surface

The tangential cracks bent. The tangential cracks near the crater, such as crack 26 in Fig. 10, were parallel to the crater cone. With the increase in the instantaneous penetration depth of the projectile, the tangential cracks (e.g., cracks 4 and 13 in Fig. 10) propagated obliquely forward and bent. With the further increase of the instantaneous penetration depth, the cracks tended to straighten (e.g., cracks 5 and 16 in Fig. 10). However, cracks 8 and 17 in Fig. 10 were affected by the target back, and formed a shear plug, causing the cracks to bend (Yankelevsky and Leibowitz, 1999).

The tangential cracks propagated asymmetrically and interacted. In Fig. 11a, cracks 1 4 propagated outward asymmetrically, and the order of reaching this cross section was as follows: crack $1>2>3>4$. In Fig. 10 a, crack 12 propagated through crack 11 , and crack 14 stopped the propagation of crack 15.

The radial cracks and the tangential cracks interacted. Approximately 8 radial cracks and some tangential spalling cracks were generated on the impact surface of the target (Fig. 10d). The tangential spalling cracks were divided by radial cracks, indicating that the tangential spalling cracks reaching the target strike surface were later than the radial cracks. A large number of radial cracks were observed on the impact surface; with an increase in the axial coordinates, the number of radial cracks propagating to the cylindrical surface of the target gradually decreased (Fig. 10b). Because radial cracks were easily stopped by tangential cracks propagating obliquely forward; for example, tangential cracks $1 \sim 4$ in Fig. 11a prevented the propagation of central radial crack 6 . The cracks at the initial stage in the pure mortar target were radial cracks, which then gradually turned into tangential cracks (Section 3.2.1); therefore, the crack near the ballistic center in Fig. 11a pointed partially toward the center and partially toward the deviation center.

The cracks in the concrete target containing coarse aggregate are shown in Figs. 12 and 13. The distributions of the cracks in the $c 1$ and c3 concrete targets were identical overall; for example, the impact surface of the target had approximately 8 radial cracks (Fig. 12b) and some tangential spalling cracks (crack 25 in Fig. 12b), and the number of radial cracks decreased with an increase in the axial coordinates (Fig. 12c). The cutting position in target c3-2 was closer to the impact surface; thus, there were still a large number of radial cracks and some radial cracks were connected to the impact surface (cracks 1, 5 and 6 in Figs. 13a and 13e). The cylindrical holes in the target edge shown in Fig. 13 were all the core holes before penetration, and both radial crack 1 and tangential crack 13 were able to propagate through the coring holes. 



Figure 12: Cracks in target c3-3 $\left(V_{0}=650 \mathrm{~m} / \mathrm{s}, P=0.702 \mathrm{~m}\right)$, (a) Section, (b) Impact surface, (c) (f) Side surface
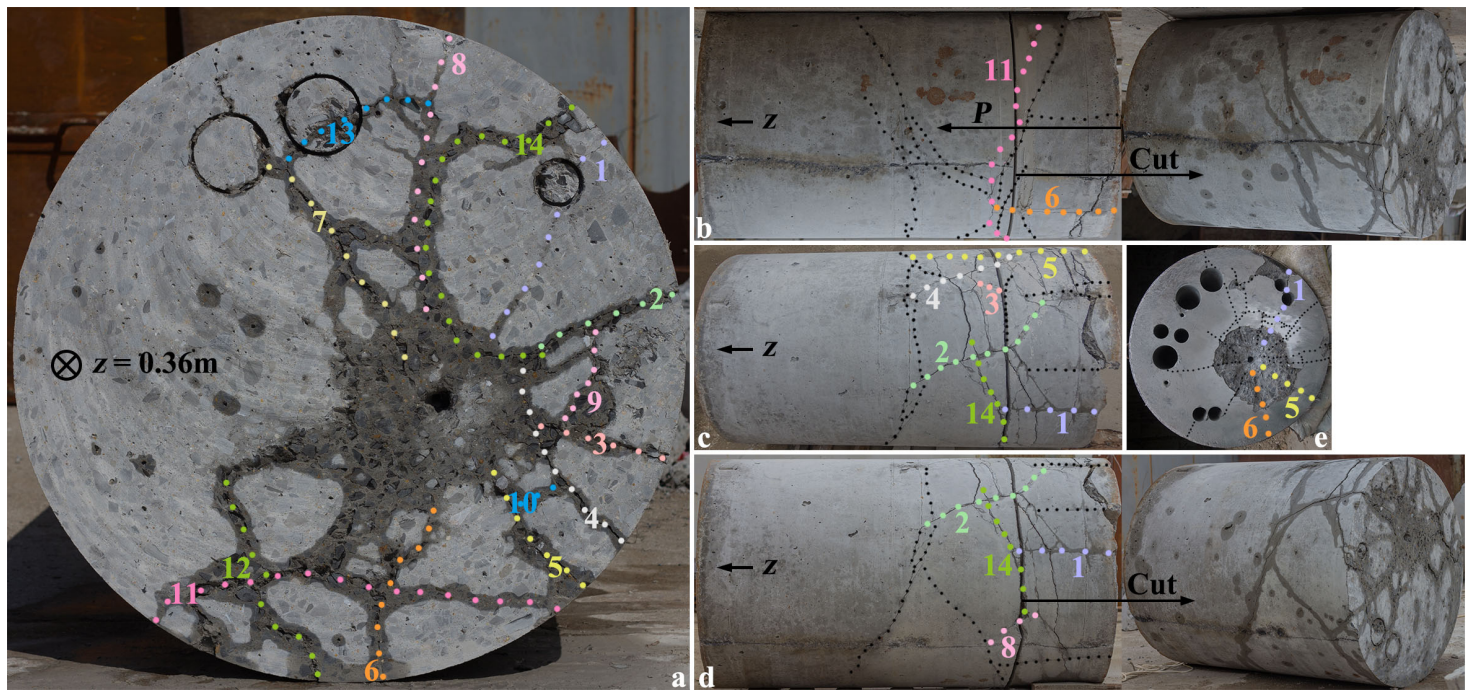

Figure 13: Cracks in target c3-2 $\left(V_{0}=653 \mathrm{~m} / \mathrm{s}, P=0.659 \mathrm{~m}\right)$, (a) Section, (b) (d) Side surface, (e) Impact surface

The addition of coarse aggregates reduced the compressibility of the concrete, and increased the dynamic tensile strength of the material, and thus increased the radius of the brittle damage zone (Figs. 10a and 12a). Near the c3-3 tunnel zone, higher instantaneous projectile velocity corresponded to stronger tangential inertial confinement of the concrete, greater range of the triaxial compressive stress state, and greater distance between the brittle damage zone and the ballistic trajectory (Fig. 12a). At the ballistic end, the velocity of the projectile approached zero, the concrete had no tangential inertial constraints, and the brittle damage zone was situated near the projectile head. However, due to the overall small radius of brittle damage zone in target c1-4, the influence of the instantaneous projectile velocity on the radius of the brittle damage zone was not obvious. Unlike the bending tangential cracks in target c1-4, the general trend of tangential cracks in target c3-3 was relatively straight. 


\subsubsection{Crack propagation model}

A diagrammatic sketch of tangential crack propagation is shown in Fig. 14. The 3D crack surface is simplified as a $2 \mathrm{D}$ crack line. The radial coordinate and axial coordinate of the crack are respectively $r(t)$ and $z_{c}[r(t)]$, which are functions of time $t$. The coordinate of the projectile head is denoted by $z_{p}[t]$. The instantaneous velocity of the projectile is $V_{p}(t)$. The propagation velocity of a crack in concrete increases with increased strain rate; during dynamic loading (strain rate $>1 \mathrm{~s}^{-1}$ ), the crack propagation velocity approaches a constant value (Pyo et al., 2016; Pyo and ElTawil, 2013).

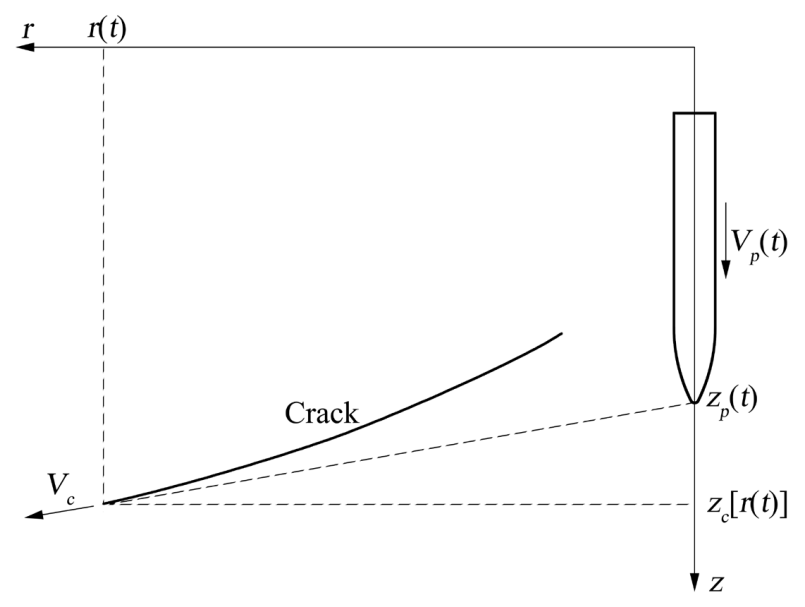

Figure 14: Diagrammatic sketch of tangential crack propagation

The constant crack velocity $V_{c}$ is expressed as:

$V_{c}=\sqrt{1+\left(\frac{d z_{c}[r(t)]}{d r(t)}\right)^{2}} \frac{d r(t)}{d t}$

The projectile nose acted on the concrete, and the stress wave propagated in a nearly hemispherical shape. The propagation velocity was much larger than the velocity of the projectile. Therefore, the reverse direction of the crack propagation direction approximately points to the projectile nose, that is:

$z_{p}(t)=z_{c}[r(t)]-r(t) \frac{d z_{c}[r(t)]}{d r(t)}$

The derivative of Eq. (2) to time $t$ is:

$V_{p}(t)=-r(t) \frac{d^{2} z_{c}[r(t)]}{d r(t)^{2}} \frac{d r(t)}{d t}$

The crack propagation velocity $V_{c}$ is large; hence, the projectile moving distance is limited during crack formation. Thus, the change in the instantaneous velocity $V_{p}(t)$ of the projectile can be ignored. Combining Eq. (1) and Eq. (3) to obtain the general expression formula of a crack gives:

$z_{c}(r)=\frac{\mathrm{e}^{\mathrm{c}_{1} r}{ }^{1-\frac{V_{p}}{V_{c}}}}{2\left(1-\frac{V_{p}}{V_{c}}\right)}-\frac{\mathrm{e}^{-c_{1}} r{ }^{1+\frac{V_{p}}{V_{c}}}}{2\left(1+\frac{V_{p}}{V_{c}}\right)}+\mathrm{c}_{2}$

where $c_{1}$ and $c_{2}$ are integral constants, and $V_{p}$ is the average instantaneous velocity of the projectile corresponding to a specific crack. 
When a projectile with a medium initial velocity deeply penetrates a concrete target, the penetration resistance can be approximated as a constant (Frew et al., 2006; Rosenberg and Dekel, 2010); thus, an approximate expression of $V_{p}$ can be obtained:

$$
V_{p}=V_{0} \sqrt{\frac{P-z_{p}}{P}}
$$

The initial velocity $V_{0}$ of a projectile and the penetration depth $P$ are known. The average coordinate $z_{p}$ of the projectile nose corresponding to the specific crack can be obtained by Fig. 10a. Projectile average instantaneous velocity $V_{p}$ corresponding to a specific crack in target c1-4 was obtained by Eq. (5), as shown in Table 3.

Table 3: Projectile average instantaneous velocity $V_{p}$ corresponding to a specific crack in target c1-4

\begin{tabular}{ccccc}
\hline Crack No. & $\mathbf{2}$ & $\mathbf{4}$ & $\mathbf{1 0}$ & $\mathbf{1 3}$ \\
\hline$V_{p}(\mathrm{~m} / \mathrm{s})$ & 594 & 494 & 581 & 488 \\
\hline
\end{tabular}

The occurrence of cracks is random, that is, the location and propagation direction of each crack is different. In the Eq. (4), c1 and $c_{2}$ represent the randomness of the crack. However, the crack propagation velocity $V_{c}$ is an essential property of the crack. Therefore, after fitting the crack using Eq. (4), for different cracks, c1 and c2 are different, but $V_{c}$ is similar.

The fitting curve is in good agreement with the experiment, as shown in Fig. 15. The cracks at four different locations have similar propagation velocities $V_{c}$ with an average of $1060 \mathrm{~m} / \mathrm{s} . V_{c}=0.41 C_{L}$, consistent with the conclusions of Zhao and Xiao (2013), indicating that the crack propagation velocity obtained in this paper is reasonable.

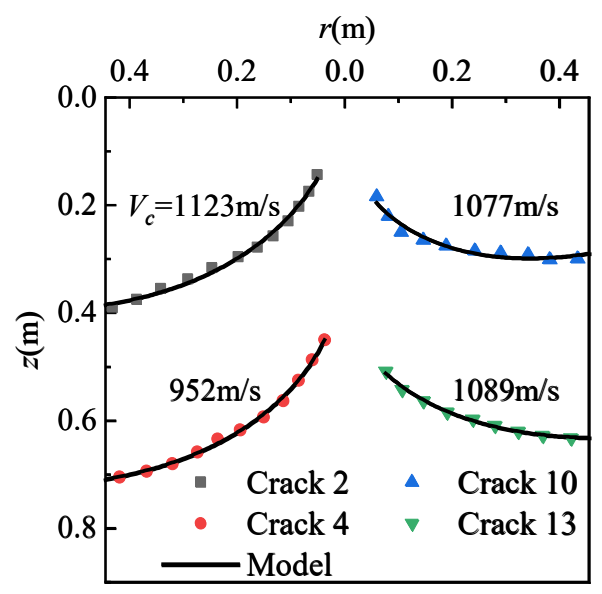

Figure 15: The tangential crack propagation law of target c1-4

When $V_{p} / V_{c}$ is close to 0 , Eq. (4) can be simplified to:

$z_{c}(r)=\frac{\mathrm{e}^{\mathrm{c}_{1}}-\mathrm{e}^{-\mathrm{c}_{1}}}{2} r+\mathrm{c}_{2}$

With a decrease in $V_{p} / V_{c}$, the crack approaches a straight line. As shown in Fig. 10a, with an increase in the instantaneous penetration depth of the projectile, $V_{p}$ decreased and $V_{c}$ did not change, and thus, the crack became increasingly straight.

According to Eq. (6), it is not possible to calculate the propagation velocity of a straight crack. For the concrete containing coarse aggregate, the cracks were straight (Fig. 12a); thus, the crack propagation velocity could not be calculated by the model proposed in this paper; but it can be predicted that the crack propagation velocity is higher than that in the pure mortar target. If the initial velocity of the projectile is further increased, the tangential cracks in the concrete target containing coarse aggregate will be bent; then, the crack propagation velocity in the concrete containing coarse aggregate at a high strain rate will be obtained. 
In this paper, the general expression formula of the crack shape under the penetrating load was given, and the crack propagation velocity was fitted by the crack shape result. However, the initial direction and position of the crack are random (affected by nose shape and instantaneous velocity of projectile, aggregate distribution and random defects), so the crack distribution in the target cannot be calculated in this paper. The crack initiation mechanism in target needs further study.

\section{CONCLUSIONS}

Experimental and theoretical studies of cracks were carried out for pure mortar targets and concrete targets containing coarse aggregate penetrated by projectile. The conclusions are as follows:

1. The crack distribution in the target was divided into a plastic damage zone and a brittle damage zone. The plastic damage zone contained microcracks and compressed pores; its density increased, and the elastic modulus and uniaxial compressive strength decreased significantly. The microcracks in the plastic damage zone were transformed by macrocracks. There were no microcracks in the brittle damage zone, and macrocracks were propagated locally. The randomly distributed coarse aggregate caused the macrocracks to become serrated.

2. The propagation laws of radial cracks and tangential cracks were obtained. At the initial stage of macrocrack propagation, the direction of the crack in the pure mortar target changed from radial to tangential, while the target containing coarse aggregate formed tangential cracks directly. At the stage of crack propagation, the tangential cracks propagated asymmetrically, and the number of radial cracks was gradually reduced due to being blocked by tangential cracks. The tangential cracks were bent in the pure mortar target and were relatively straight in target containing coarse aggregate. The radius of brittle damage zone in the target containing coarse aggregate was larger than that in pure mortar target.

3. A tangential crack propagation model was established, and the general formula of the crack was obtained. The crack propagation velocity in the pure mortar target was further obtained, which was $1060 \mathrm{~m} / \mathrm{s}$. The developed model showed that tangential cracks tended to be straight when projectile instantaneous velocity was much smaller than the crack propagation velocity. It is inferred that the crack propagation velocity in concrete containing coarse aggregate is higher than that in pure mortar target.

\section{ACKNOWLEDGMENT}

This work was supported by the National Natural Science Foundation of China [grant number 11602111].

\section{References}

Beissel S. R., Johnson G. R. (2000). An abrasion algorithm for projectile mass loss during penetration. Int J Impact Eng 24(2): 103-116.

Forquin P., Arias A., Zaera R. (2008). Role of porosity in controlling the mechanical and impact behaviours of cement-based materials. Int J Impact Eng 35(3): 133-146.

Forquin P., Hild F. (2010). A Probabilistic Damage Model of the Dynamic Fragmentation Process in Brittle Materials. Adv Appl Mech 44:1-72.

Forquin P., Sallier L., Pontiroli C. (2015). A numerical study on the influence of free water content on the ballistic performances of plain concrete targets. Mech Mater 89:176-189.

Frew D. J., Forrestal M. J., Cargile J. D. (2006). The effect of concrete target diameter on projectile deceleration and penetration depth. Int J Impact Eng 32(10): 1584-1594.

Kanninen M. F., Popelar C. H. (1987). Advanced fracture mechanics. Beijing: Beijing Institute of Aeronautics Press.

Lai J., Guo X., Zhu Y. (2015). Repeated penetration and different depth explosion of ultra-high performance concrete. Int J Impact Eng 84:1-12.

Li Q. M., Reid S. R., Wen H. M., et al. (2005). Local impact effects of hard missiles on concrete targets. Int J Impact Eng 32(1):224-284. 
Lian B., Jiang J. W., Men J. B., et al. (2010). Simulation analysis on law of penetration of long-rod projectiles with high speed into concrete. Chin J High Press Phy 24(5):377-382.

Pereira L. F., Weerheijm J., Sluys L. J., et al. (2018). Simulation of compaction and crushing of concrete in ballistic impact with a new damage model. Int J Impact Eng 111: 208-221.

Pyo S., Alkaysi M., El-Tawil S. (2016). Crack propagation speed in ultra high performance concrete (UHPC). Constr Build Mater 114: 109-118.

Pyo S., El-Tawil S. (2013). Crack velocity-dependent dynamic tensile behavior of concrete. Int J Impact Eng 55: 63-70.

Rosenberg Z., Dekel E. (2010). The deep penetration of concrete targets by rigid rods-revisited. Int J Protective Struct 1(1): 125-144.

Sovják R., Vavřiník T., Zatloukal J., et al. (2015). Resistance of slim UHPFRC targets to projectile impact using in-service bullets. Int J Impact Eng 76: 166-177.

Tai Y. S. (2009). Flat ended projectile penetrating ultra-high strength concrete plate target. Theor Appl Fract Mec 51(2): 117128.

Wu C., Li W. B., Shen X. J. (2018). Study of the anti-penetration performance of concrete with different coarse aggregate content. Lat Am J Solids Stru 15(6).

Wan F., Jiang Z., Tan Q., et al. (2016). Response of steel-tube-confined concrete targets to projectile impact. Int J Impact Eng 94: 50-59.

Wu H. J., Huang F. L., Wang Y. N., et al. (2012). Experimental investigation on projectile nose eroding effect of high-velocity penetration into concrete. Acta Armamentarii 33(1): 48-55.

Yankelevsky D. Z., Leibowitz O. (1999). Punching shear in concrete slabs. Int J Mech Sci 41(1):1-15.

Zhao X. J., Xiao C. (2013). Experimental Investigation of reinforced concrete medium crack growth rate under explosion impact load. Chin J Expl Propell 36(1):55-58. 